\title{
Helminthes parasites d'animaux sauvages au Sénégal
}

(Deuxième note)

\author{
Syncuaria (Skrjabinocara) leptoptili Gedoelst, 1916, \\ Syncuaria (Skrjabinocara) bressoui n. sp. (Nematoda) \\ et Balfouria monogama Leiper, 1908 (Trematoda), \\ chez Leptoptilus crumenifer (Less.) (Marabou stork) \\ par Simon GRETILLAT \\ Laboratoire de Parasitologie et de Mycologie (Pr M. LARIVIÈRE), U.E.R. des Cordeliers, \\ 15 , rue de l'Ecole-de-Médecine, F 75 - Paris-6 ${ }^{\circ}$
}

\begin{abstract}
Résumé
Un important lot d'Acuarides récolté dans la sous-muqueuse de l'estomac (ventricule succenturié) d'un Leptoptilus crumenifer (Marabout) abattu au Sénégal permet l'étude détaillée de Syncuaria leptoptili (Gedoelst, 1916) que certains caractères morphologiques rangent dans le sous-genre Skrjabinocara Kurashwili, 1941, et la description d'un nouveau Dispharage de cette cigogne, Syncuaria (Skrjabinocara) bressoui $\mathrm{n}$. $\mathrm{sp}$. Le Trématode Balfouria monogama Leiper, 1908, parasite de l'intestin de cet oiseau, est signalé pour la première fois au Sénégal.
\end{abstract}

\section{Summary}

An important lot of Acuaridae found in the stomach mucous membrane (succenturions ventricle) of a Leptoptilus crumenifer (Marabout), shot in Senegal, allowed a detailed study of Syncuaria leptodactili (Gedoelst, 1916), (which several morphological features set in the sub-genus Skrjabinocara Kurashwili, 1941), and the description of a new Dispharage of this stork, Syncuaria (Skrjabinocara) bressoni n. sp. The Trematoda Balfouria monogama Leiper, 1908, parasite of this bird intestine is mentionned for the first time in Senegal. 
Au cours d'une partie de chasse dans la région du delta du fleuve Sénégal, en décembre 1966, nous avons eu la chance de récolter dans la muqueuse du ventri-

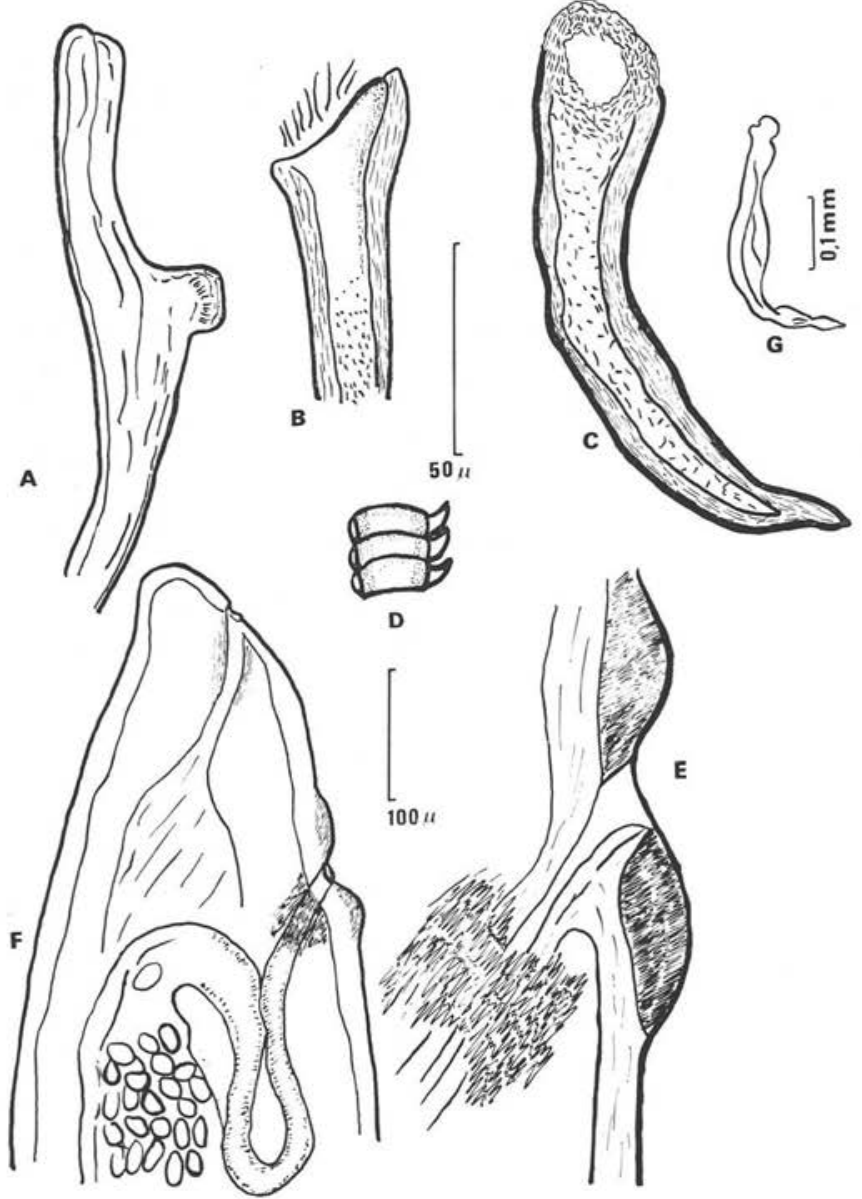

Fig. 1. - Syncuaria (Skrjabinocara) leptoptili (Gedoelst, 1916). A. Extrémité distale du spicule gauche. B. Extrémité antérieure ou tête du spicule gauche. C. Spicule droit. D. Cordon céphalique (détail). E. Orifice vulvaire. F. Extrémité postérieure de la femelle. G. Syncuaria hargilae (Baylis et Daubney, 1922). Spicule droit (d'après Baylis et Daubney, 1922)

cule succenturié d'un Marabout (Leptoptilus crumenifer Less.), une centaine de spirures appartenant au genre Syncuaria Gilbert, 1927 et que certains caractères morphologiques (présence de deux fins cordons cuticulaires latéraux allant des dei- 
rides à l'extrémité postérieure du corps), placent dans le sous-genre Skrjabinocara Kurashwili, 1941. Chez le même oiseau, plusieurs dizaines de trématodes de l'espèce Balfouria monogama Leiper, 1908, étaient accolés par paires et comme enkystés dans la paroi du gros intestin.

Ordre des Spiruridae Diesing, 1861.

Famille des Acuariidae Seurat, 1913.

Genre Syncuaria Gilbert, 1927.

Sous-genre Skrjabinocara Kurashwili, 1941.

Espèce : Syncuaria (Skrjabinocara) leptoptili (Gedoelst, 1916).

Un peu plus de la moitié du lot de spirurides (environ une cinquantaine d'exemplaires) appartient à cette espèce. La qualité du matériel récolté nous permet d'apporter quelques précisions concernant certains caractères morphologiques. Nous les donnons sous forme de tableau comparatif avec ceux trouvés par Gedoelst en 1916 et Gendre en 1919.

C'est à partir d'un prélèvement en provenance de Dolo (Congo-Kinshasa) récoltés par le docteur Rovere dans l'estomac d'un Marabout (Leptoptilus crumenifer) que Gedoelst a décrit cette espèce. Malheureusement le lot examiné ne renfermait que des femelles.

Trois ans plus tard, Gendre (1919), en étudiant une collection d'helminthes rassemblée par le docteur Bouet en Afrique occidentale française trouve un tube contenant 33 femelles et 1 mâle de spirures récoltés à Bodjécali (Dahomey) en janvier 1910 chez un Marabout.

Gendre identifie ces nématodes comme appartenant à l'espèce de Gedoelst et décrit le mâle d'Echinuria leptoptili.

Baylis et Daubney en 1922 signalent ce «Dispharage» aux Indes chez Leptoptilus dubius à Calcutta. L'année suivante, en comparant leur matériel avec la description de Gendre (1919), ils considèrent les spirures de l'estomac de L. dubius comme différents d'E. leptoptili et l'appellent Echinuria hargilae du nom local donné au marabout de l'Inde «Hargila ».

Gendre en 1919 signale que son matériel est légèrement détérioré, ce qui ne l'empêche pas de dire: «J'ai pu constater de cette manière quelques différences qui tiennent vraisemblablement à ce que les exemplaires observés par le savant professeur belge n'étaient pas dans un parfait état de conservation. » Tout ceci pour tenter d'expliquer les différences dans certaines dimensions et particulièrement dans celles des cordons céphaliques.

La description du mâle d'E. leptoptili par Gendre correspond bien dans l'ensemble aux 26 exemplaires que nous avons à notre disposition. Cependant, si les dimensions du spicule gauche données par cet auteur sont identiques à celles de nos exemplaires, «la petite protubérance latérale à sommet mousse, légèrement recourbée en arrière », qui donne à l'extrémité du spicule, «l'aspect d'un harpon quand on le regarde par la face dorsale », est chez certains exemplaires tellement peu proéminente 
qu'il est difficile de parler de «harpon », mais plutôt d'un épaississement du spicule suivi d'une torsion terminale.

Les chiffres donnés par Gedoelst et par Gendre au sujet des cordons céphaliques et des portions de l'œsophage montrent bien que tous les deux n'avaient à leur disposition que du matériel plus ou moins bien conservé. Gendre parle de cordons festonnés alors que tous les spécimens en notre possession ont des cordons presque rectilignes. D'autre part, si l'ornementation des cordons est un caractère valable, la distance de leur anastomose postérieure à l'extrémité céphalique est trop variable pour être un bon critère d'espèce.

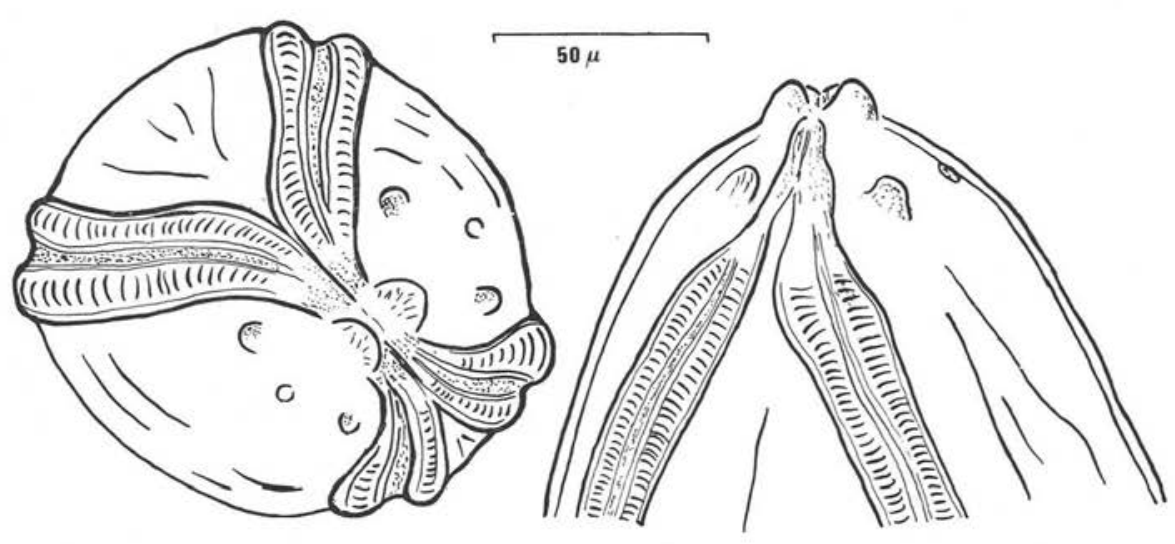

Fig. 2. $-S$. leptoptili, tête vue apicale et vue de profil

La structure céphalique examinée en vue apicale permet de constater l'existence de deux pseudo-lèvres à bords semi-circulaires, encadrées par quatre petites lèvres et une paire d'amphides.

$\mathrm{Si}$ Gendre parle de deirides à peine visibles, Gedoelst les voit sous forme de papilles coniques faiblement proéminentes, alors que ce sont de petits éléments globuleux de $3,5 \mu$ de diamètre qui examinés à un très fort grossissement apparaissent bicuspides avec deux petites pointes mousses de $1,5 \mu$ de longueur.

Quant à la description que donne Gedoelst pour la partie postérieure de la femelle: "le relèvement de la queue vers la face dorsale, la dilatation de la face ventrale en avant de la vulve », ne sont que la protubérance terminale de la queue implantée dorsalement, et le sphincter périvulvaire très développé, donnant à l'extrémité postérieure cette allure obtuse que l'auteur décrit en parlant du \& recouvrement de cet organe (la vulve) masquant en partie l'atténuation postérieure du corps ». Cette description imagée nous semble exacte, mais il est regrettable que Gedoelst n'ait illustré son texte d'aucun dessin ou schéma. 
Un examen minutieux à très fort grossissement permet de voir deux cordons cuticulaires latéraux constitués par une série de plaques minces et subovalaires, juxtaposées tout au long de chaque ligne latérale du corps de l'helminthe. Partant un peu en arrière de chaque deiride où il est peu distinct et a l'apparence d'un sillon, ce cordon très nettement visible quoique peu saillant, dans sa partie moyenne, se termine un peu en avant de l'extrémité postérieure; c'est un caractère du sous-genre Skrjabinocara Kurashwili, 1941, qui est le synonyme du sous-genre Chordocephalus

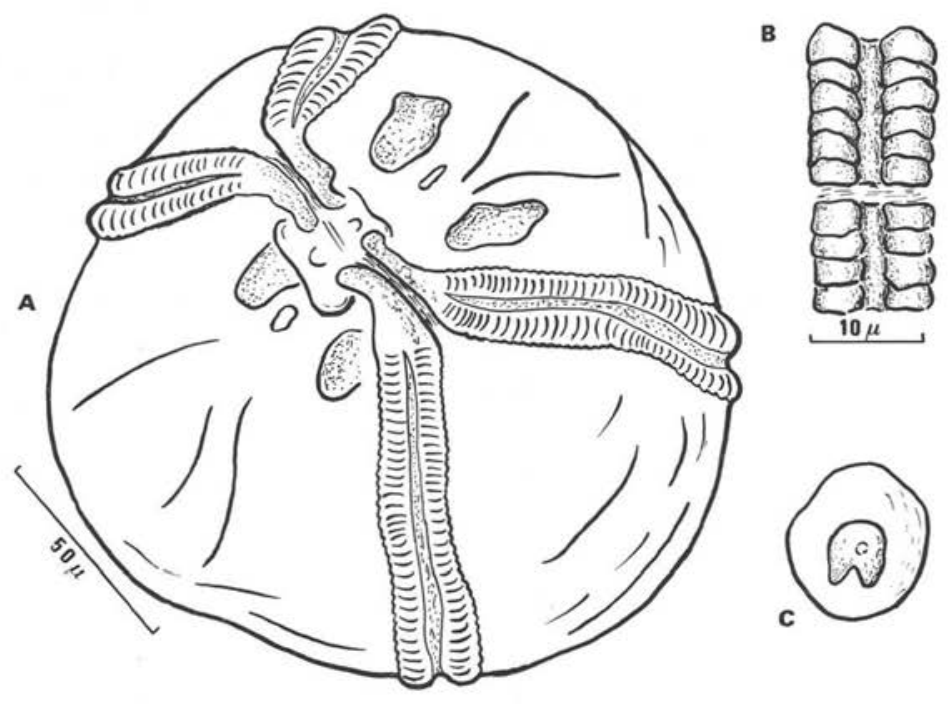

FIG. 3. - Syncuaria (Skrjabinocara) bressoui n. sp. A. Tête, vue apicale. B. Cordon céphalique (vue au niveau de la jonction postérieure). C. Deiride

Alegret, 1941. Tous deux sous-genres du genre Syncuaria Gilbert, 1927 auquel appartient l'espèce que Gedoelst a décrite sous le nom d'Echinuria leptoptili qui devient : Syncuaria (Skrjabinocara) leptoptili (Gedoelst, 1916).

\section{[Syncuaria (Skrjabinocara) leptoptili], n. sp.}

Cet important lot de spirures renfermait également un certain nombre d'exemplaires (22 mâles et 35 femelles) appartenant aussi au genre Syncuaria et au sousgenre Skrjabinocara, mais différents de l'espèce précédente.

Habirus : Un peu plus court que $S$. leptoptili, le mâle de ce Dispharage, blanc nacré à l'état frais, a une queue très enroulée sur elle-même et des ailes caudales très développées en longueur et en largeur. La femelle a une extrémité postérieure très obtuse avec une vulve placée en position postérieure juste en avant de l'anus. Examinée à 
ia loupe, la partie postérieure de la femelle apparaît comme taillée en «biseau » très court dont la surface de section serait ornée de deux petites élevures correspondant aux bourrelets sphinctoriaux entourant l'orifice vulvaire:

MÂLE :

Longueur moyenne: $7,5 \mathrm{~mm}$.

Largeur moyenne maximum : $200 \mu$.

Cuticule finement striée, distance interstries : $4 \mu$.

L'extrémité céphalique est ornée de deux pseudo-lèvres à bords épais légèrement infléchis en leur milieu; quatre énormes papilles latérales situées tout contre i'origine des cordons céphaliques encadrent deux petites amphides peu visibles.

Les cordons céphaliques (deux paires), parcourent les parties latérales de l'extrémité antérieure sans festons apparents; leur largeur moyenne est de $13 \mu$ et leur épaisseur d'environ $7 \mu$. Ce n'est qu'au niveau de leur anastomose postérieure sans

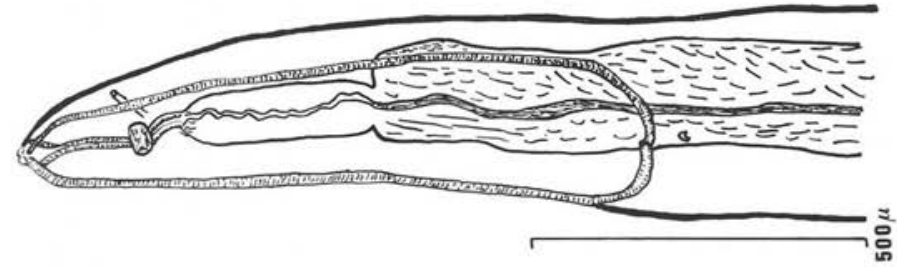

FIG. 4. - S. bressoui n. sp., extrémité antérieure de la $q$

récurrence qu'ils deviennent un peu plus étroits. A ce niveau situé à environ 690 à $700 \mu$ de l'extrémité céphalique, ils s'anastomosent en laissant cependant entre eux une petite solution de continuité. Ce sont deux bandes striées parallèles séparées par un intervalle continu lisse, étroit et plus foncé, avec un bord interne orné de petites épines.

Deux deirides bicuspides à base globuleuse sont placées au centre d'une aire circulaire claire de $18 \mu$ de diamètre. Chaque deiride a $6 u$ de diamètre et se trouve à environ $50 \mu$ en arrière de l'anastomose des cordons céphaliques. Un cordon cuticulaire de $7 \mu$ de largeur, très mince et à bords crénelés prend naissance à $50 \mu$ environ en arrière de chaque deiride, parcourt le corps en position latérale pour se terminer un peu en avant de l'extrémité postérieure qui est très fortement enroulée sur elle-même. Elle est pourvue de deux ailes caudales longues et larges $1,350 \mathrm{~mm}$ de long et à bords festonnés. Queue de 100 à $120 \mu$ de long. Neuf paires de papilles caudales: 4 précloacales et 5 postcloacales; les quatre dernières sont groupées par 2 , respectivement au milieu de la queue et au niveau de son quart postérieur.

Le spicule gauche mesure 390 à $400 \mu$; son extrémité distale est filiforme et striée. C'est une baguette légèrement incurvée à parois chitinisées avec base légèrement élargie et un renflement médian placé à environ 100 à $130 \mu$ de la base. Le 
spicule droit a 95 à $100 \mu$ de longueur ; recourbé sur lui-même, il est très légèrement chitinisé et son extrémité distale terminée par deux pointes est nettement coudée à angle droit par rapport à la partie moyenne du spicule.

Le tube intestinal comprend un pharynx à parois striées mesurant $220 \mu$, un œsophage musculaire de $240 \mu$ et un œsophage glandulaire de $1,78 \mathrm{~mm}$ de longueur.

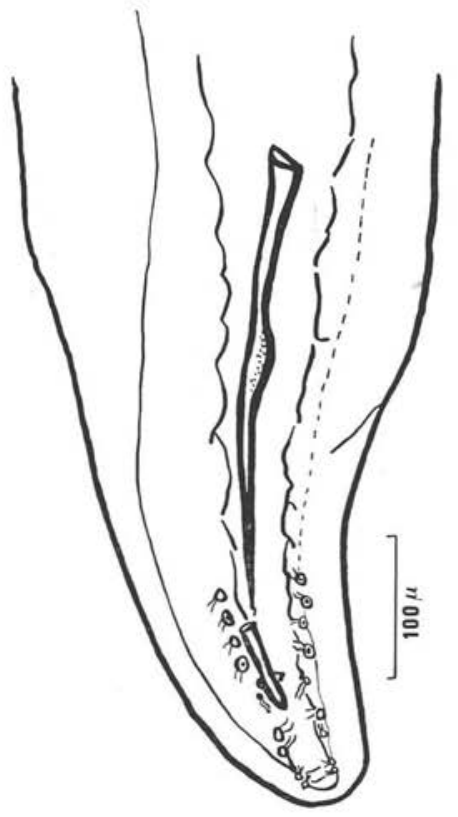

FIG. 5. - S. bressoui, extrémité postérieure du mâle
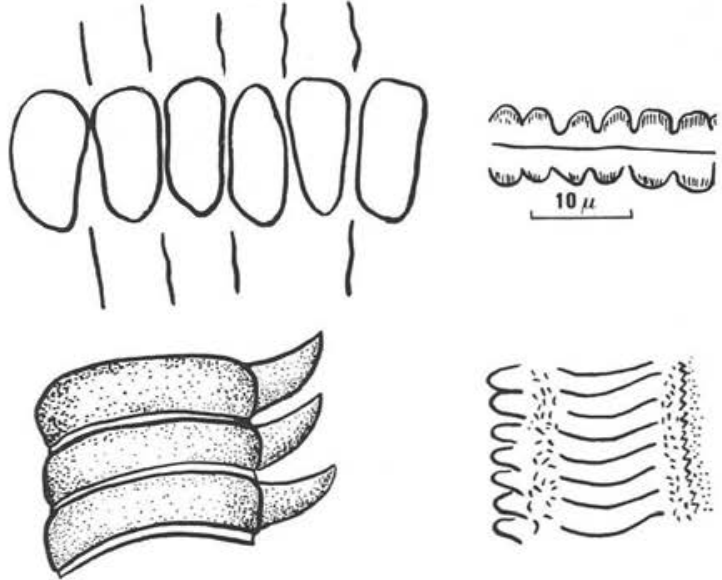

L'anneau nerveux et le pore excréteur se trouvent respectivement à 145 et $350 \mu$ de l'extrémité céphalique.

\section{FEMELLE :}

Longueur: 8,5 à $9,3 \mathrm{~mm}$.

Largeur maximum (moyenne) : $350 \mu$;

beaucoup plus trapue que le mâle mais de taille sensiblement égale au moins macroscopiquement. Cuticule striée, distance interstries : $6 \mu$.

Longueur des cordons céphaliques: $950 \mu$ environ avec une largeur maximum de $18 \mu$ et une épaisesur de $8 \mu$; non festonnés et presque rectilignes. Deirides à $50 \mu$ en arrière de l'anastomose des cordons. Extrémité postérieure très obtuse. Queue très courte : $60 \mu$. Vulve à $120 \mu$ de l'extrémité du ver qui est arrondie. Un bourrelet 


\begin{tabular}{|c|c|c|c|c|c|c|c|c|c|c|c|c|c|c|c|c|c|c|c|}
\hline 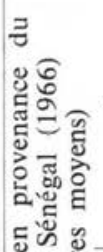 & ro & $\begin{array}{l}\varepsilon \\
\text { है } \\
\infty \\
\sigma \\
\approx \\
\text { ? }\end{array}$ & $\begin{array}{l}= \\
8 \\
\text { i } \\
\approx \\
0 \\
\end{array}$ & $\begin{array}{l}= \\
n \\
0 \\
\vdots \\
\vdots \\
\infty\end{array}$ & 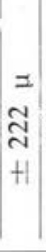 & $\begin{array}{l}7 \\
0 \\
0\end{array}$ & $\begin{array}{l}\text { E } \\
\text { ह } \\
\text { ปे } \\
\text { ci }\end{array}$ & $\begin{array}{l}1 \\
\text { 용 }\end{array}$ & $\begin{array}{l}= \\
\end{array}$ & $\begin{array}{l}\underline{E} \\
\Xi \\
\underline{0} \\
=\end{array}$ & $\begin{array}{l}= \\
=\end{array}$ & $\begin{array}{ll}z & = \\
2 & 0\end{array}$ & $\begin{array}{l}E \\
\Xi \\
\underline{-}\end{array}$ & $\begin{array}{l}= \\
n \\
\Xi\end{array}$ & & & & 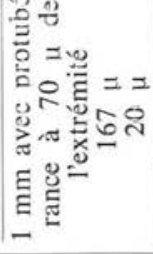 & 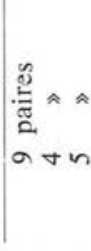 \\
\hline 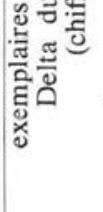 & ot & 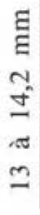 & 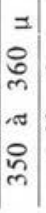 & 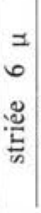 & $\begin{array}{l}= \\
\text { ปे }\end{array}$ & $\begin{array}{l}\text { छ } \\
\text { ह } \\
-\end{array}$ & $\begin{array}{l}\text { E } \\
\text { ह } \\
\text { n }\end{array}$ & $\begin{array}{l}7 \\
8 \\
\text { సิ }\end{array}$ & $\begin{array}{l}1 \\
0 \\
\tilde{\lambda}\end{array}$ & $\begin{array}{l}E \\
\Xi \\
\sim \\
-1 \\
+1\end{array}$ & $\begin{array}{l}\simeq \\
\simeq\end{array}$ & $\begin{array}{l}z= \\
m=\end{array}$ & $\begin{array}{l}E \\
\Xi \\
n \\
-\end{array}$ & $=$ & $\begin{array}{l}= \\
\stackrel{\sim}{\sim} \\
\stackrel{\sim}{m}\end{array}$ & 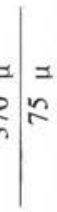 & 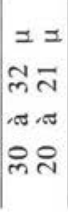 & & \\
\hline 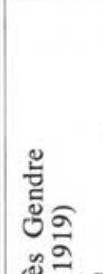 & ${ }^{\circ}$ & $\begin{array}{l}\text { E } \\
\text { E } \\
n \\
n \\
0\end{array}$ & $\begin{array}{l}= \\
8 \\
\text { i }\end{array}$ & 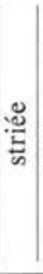 & $\begin{array}{l}z \\
0 \\
2\end{array}$ & $\begin{array}{l}z \\
0 \\
\tilde{y}\end{array}$ & 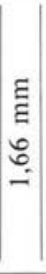 & & & $\begin{array}{l}= \\
\frac{0}{6}\end{array}$ & & & & $\begin{array}{l}= \\
8 \\
8\end{array}$ & & & & 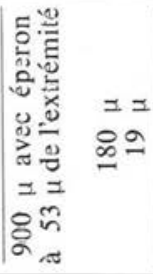 & 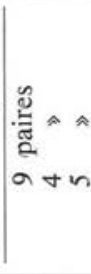 \\
\hline$\tau$ & of & $\begin{array}{l}\infty \\
\infty \\
= \\
\approx \\
\approx \\
\approx \\
\approx\end{array}$ & $\begin{array}{l}= \\
i \\
\end{array}$ & & $\begin{array}{l}= \\
\text { స్ } \\
\text { N }\end{array}$ & $\begin{array}{l}= \\
0 \\
\infty \\
+\end{array}$ & $\begin{array}{l}E \\
\text { है } \\
\text { गे } \\
\text { तi }\end{array}$ & $z^{2}$ & & $\begin{array}{l}E \\
\text { E } \\
\hat{\sigma} \\
0\end{array}$ & & & & & 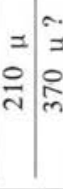 & $\begin{array}{l}= \\
= \\
\infty\end{array}$ & & & \\
\hline 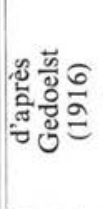 & ot & 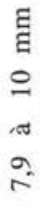 & $\begin{array}{l}= \\
0 \\
\text { f }\end{array}$ & 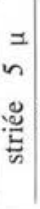 & $\begin{array}{l}1 \\
8 \\
8\end{array}$ & 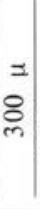 & 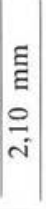 & & & $\begin{array}{l}\text { E } \\
\text { ڤે } \\
\dot{\theta}\end{array}$ & $\begin{array}{l}1 \\
6\end{array}$ & & & $\begin{array}{l}= \\
\approx \\
\approx \\
\approx\end{array}$ & 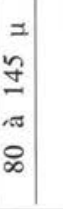 & & $\begin{array}{l}71 \\
\text { min }\end{array}$ & & \\
\hline
\end{tabular}

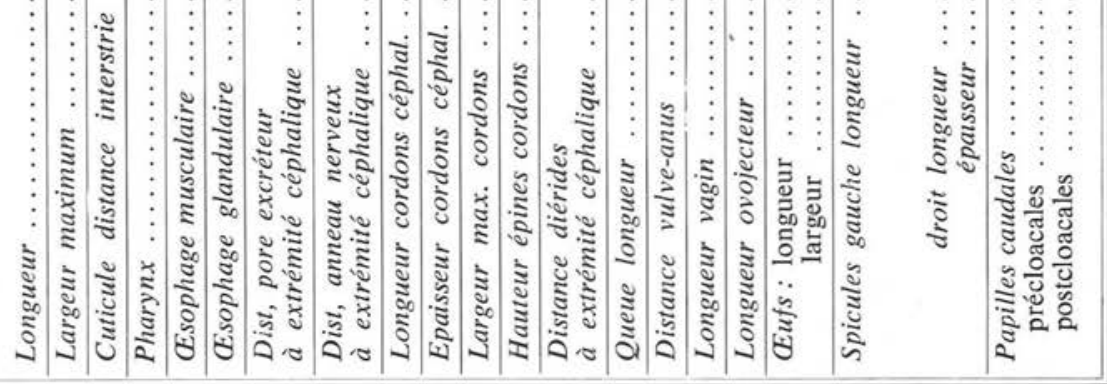


cuticulaire entoure l'orifice vulvaire à la manière de deux lèvres donnant à cette région un aspect boursouflé caractéristique. Un ovojecteur de $180 \mu$ précède la vulve. Le vagin mesure 5 à $600 \mu$, les anses utérines atteignent le point de jonction de l'œsophage glandulaire avec l'intestin. Eufs de 40 à $44 \mu$ sur 16 à $17 \mu$.

Pharynx de 250 à $270 \mu$; œsophage musculaire très court, 285 à $300 \mu$; œsophage glandulaire: $2,30 \mathrm{~mm}$.

Anneau nerveux et pore excréteur respectivement à $175 \mu$ et $465 \mu$ de l'extrémité céphalique.

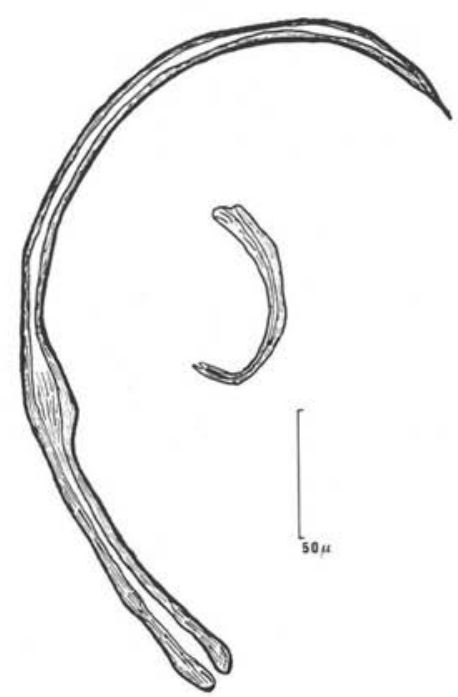

FIG. 7. $-S$, bressoui. Spicules

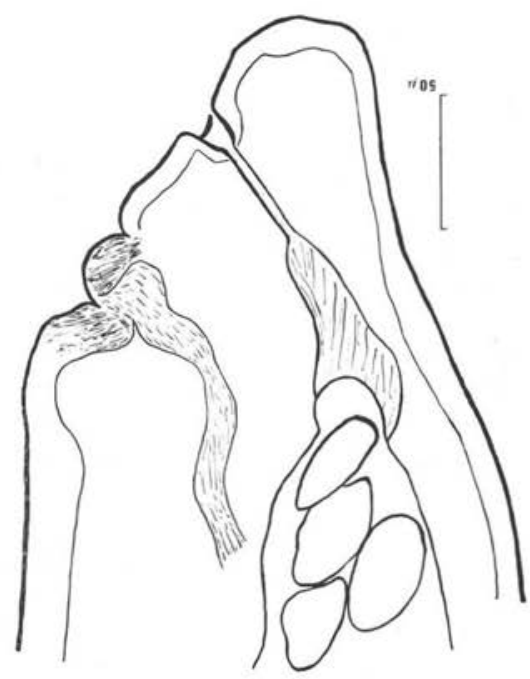

FIG. 8. $-S$. bressoui extrémité postérieure de la femelle avec détail de l'orifice vulvaire

\section{Discussion.}

Un Acuaride du Marabout Syncuaria hargilae est décrit en 1922 par Baylis et Daubney. Le spicule droit de ce Nématode est caractéristique et très différent de celui de notre espèce. Nous citons : \& It lies roughly at right angles to the long axis at the right spicule measures $0.19-0.21 \mathrm{~mm}$ in lenght, and is twisted and flanged. The root of the spicule is expended. Its edges are finely serrated in the part towards the tip. » D'autre part, la longueur et la forme du spicule gauche, les dimensions de la queue, la position de la vulve chez la femelle ainsi que l'emplacement et la morphologie des deirides sont autant de caractères qui éloignent $S$. hargilae de l'espèce que nous venons de décrire.

Les autres espèces de Syncuaria appartenant au sous-genre Skrjabinocara déjà décrites: S. squamatum (Linst., 1883), S. bukleyi Ali, 1957, S. schikobalovi Guschanskaia, 1950, S. viktori Guschanskaia, 1950, S. rostombekovi Kuraschwili, 1941, 
et $S$. parvepapillata Mack, 1962, sont toutes des parasites de Phalacrccorax (Cormoran) divers de Russie ou des Indes, à l'exception de l'avant-dernière décrite d'un aigle et de la dernière trouvée chez Ciconia ciconia en Europe centrale.

En conséquence nous considérons l'espèce que nous venons de décrire comme nouvelle et l'appellerons: Syncuaria (Skrjabinocara) bressoui n. sp. en la dédiant à notre maître, M. le Professeur Clément Bressou, directeur honoraire de l'Ecole nationale vétérinaire de Maisons-Alfort.

HôTe : Leptoptilus crumenifer (Less.) (Marabout).

LOCALISATION : sous-muqueuse du ventricule succenturié.

Provenance: Savoigne, bord de la rivière Lampsar, région du delta du fleuve Sénégal.

DATE : 26 décembre 1966.

Trematoda, famille des Echinostomidae Poche, 1926.

Sous-famille des Balfourinae Yamaguti, 1938.

Genre Balfouria Leiper, 1908.

Espèce : Balfouria monogama Leiper, 1908.

L'intestin de ce même Marabout était très fortement parasité (plusieurs dizaines d'exemplaires) par ce Trématode enkysté par paires, face ventrale contre face ventrale dans la paroi du tube digestif donnant à ce dernier un aspect monoliforme et induré. C'est à notre connaissance la première fois que cet helminthe est signalé au Sénégal.

\section{Bibliographie}

ALI (S. M.), 1957. - Studies on the nematode parasites of fishes and birds found in Hyderebad State. Indian J. Helminth., VIII, (1) : 1-83.

Baylis (H. A.) and Daubney (R.), 1923. - Report on the parasitic nematodes in the collection of the zoological Survey in India. Mem. Ind. Mus., VII, (4) : 263-347.

- - 1923. - Note on a new species of Acuaria (Nematoda) from the adjudant stork. Ann. Mag. Nat. Hist., ser. 9, XII : 95-96.

Gedoelst, 1916. - Note sur la faune parasitaire du Congo Belge. Rev. Zool. Afric., V, (1): 52-53.

Gendre, 1919. - Description du mâle d'Echinuria leptoptili, Dispharage parasite du Marabout. Proc. verb. Soc. Linn. Bordeaux, LXXI, (1 et 2) : 45-49.

GuschansKaia (L. K.), 1950. - New nematodes of the genus Skrjabinocara Kuraschwili, 1941. Trudy Gelmint. Lab. Akad. Nauk SSSR, III : 191-198.

KURASHWILI (B. E.), 1941. - Studies on the helminthofauna of Georgian birds. Trudy Zool. Inst. Akad. Nauk SSSR, IV : 53-100.

SAIDOV (Y.S.), 1954. - New species of parasitic worms of ichthiophagous birds in Daghestan. Trudy Gelmint. Lab. Akad. Nauk SSSR, VII : 265-273.

Remerciements: Nous sommes reconnaissant à M. le Professeur A.-G. Chabaud du Muséum d'histoire naturelle de Paris pour toute son aide, ses critiques et conseils au sujet de ce travail ainsi qu’à M. le Professeur M. Larivière de la Faculté de Médecine de Paris de nous avoir permis de le réaliser dans un de ses laboratoires. 